\title{
ON REAL SINGULARITIES OF LEGENDRE EXPANSIONS
}

GILBERT WALTER

1. Introduction. In a recent paper, Gilbert and Howard [G-H-1] have studied the relation between the singular points of the function given by the power series $\sum a_{n} \xi^{n}$ and the function given by the Sturm-Liouville series $\sum a_{n} v_{n}(t)$ when both series have the same coefficients and the former has a radius of convergence greater than 1 . Nehari [N-1] had previously done the same for the case of the Legendre series.

The procedure used in both cases depends on the fact that the series converges to a function holomorphic in a complex neighborhood of a real interval. If the function has a singular point in this interval the procedure fails. But many important Sturm-Liouville and Legendre series converge to functions with real singular points. One would like to associate with the series an analytic function which preserves the relation between these singular points and the singular points of the function given by the associated power series.

In this paper we show that this is possible for the Legendre series. In fact, the series $\sum a_{n}(i / \pi) Q_{n}(z)$, where the $Q_{n}$ are the Legendre functions of the second kind, converges to the right sort of analytic function. This analytic function we find to be the analytic representation of the distribution to which the Legendre series $\sum a_{n} P_{n}(t)$ converges.

The proof of these statements uses a modification of the "multiplications of singularities" technique used by the authors cited above. The extension of this result to other orthogonal systems of polynomials is possible $(\$ 4)$.

2. Properties of $P_{n}$ and $Q_{n}$. We summarize here some of the properties of the Legendre functions of the first and second kind which we shall need. An expression relating the two which is found in Szegö [S-1, p. 75] is

$$
Q_{n}(z)=\frac{1}{2} \int_{-1}^{1} \frac{P_{n}(t)}{z-t} d t .
$$

The same source tells us that $Q_{n}$ is holomorphic in the complex plane cut along $[-1,1]$ and that $Q_{n}$ asymptotically satisfies

$$
Q_{n}(x) \simeq x^{-n-1} \quad \text { as } x \rightarrow \infty .
$$

Presented to the Society, September 1, 1967; received by the editors July 31, 1967. 
From the generating function for $P_{n}(t)$ given on p. 69 of [S-1] we calculate that

$$
\sum_{n=0}^{\infty} P_{n}(t) \xi^{-n}=\xi\left(1-2 t \xi+\xi^{2}\right)^{-1 / 2}, \quad|\xi|>1,-1<t<1
$$

and

$$
\begin{aligned}
\sum_{n=0}^{\infty}(2 n+1) P_{n}(t) \xi^{n}=\left(1-\xi^{2}\right)\left(1-2 t \xi+\xi^{2}\right)^{-3 / 2}, & \\
& |\xi|<1,-1<t<1 .
\end{aligned}
$$

We use formula (3) together with (1) to prove the

LEMMA. The function given by the convergent series $\sum Q_{n}(z) \xi^{-n}$ for $|\xi| \geqq 1$ and $|z|$ sufficiently large may be continued to an analytic function $K(z, \xi)$ whose only possible singular points in the $z$ plane and the set where $|\xi| \geqq 1$ are at $z= \pm 1$ and $z=\frac{1}{2}(\xi+1 / \xi)$.

We have, for large $|z|$ and $|\xi|>1$,

$$
\begin{aligned}
K(z, \xi) & =\sum_{n=0}^{\infty} Q_{n}(z) \xi^{-n}=\frac{1}{2} \sum_{n=0}^{\infty} \int_{-1}^{1} \frac{P_{n}(t)}{z-t} d t \xi^{-n} \\
& =\frac{1}{2} \int_{-1}^{1} \frac{1}{z-t} \frac{\xi}{\left(1-2 t \xi+\xi^{2}\right)^{1 / 2}} d t,
\end{aligned}
$$

since $\sum P_{n}(t) \xi^{-n}$ converges uniformly for $t \in[-1,1]$. This last representation allows us to deduce that $K(z, \xi)$ has singular points at most at $z= \pm 1$ and at the conmmon singular points of $(t-z)^{-1}$ and $\xi\left(1-2 t \xi+\xi^{2}\right)^{-1 / 2}$. This last statement follows from the "Hadamard argument." Briefly, it goes like this: We consider the integral in (5) as a contour integral. Then keeping the end points fixed, we may deform the path of integration without changing the value of the integral provided the singular points of the integrand are avoided. We may allow $z$ to roam around the complex plane, always deforming the contour to avoid the point $t=z$. This works until $z$ approaches another singular point of the integrand in which case the contour becomes trapped, and that value of $z$ becomes a candidate for a singular point of $K(z, \xi)$. This is the value given in the conclusion of the lemma.

The same argument has been used by both Howard and Gilbert [G-H-1] and Nehari [N-1] to reach similar conclusions. We use it again below and shall refer to it simply as the "Hadamard Argument."

We shall require one more formula relating $P_{n}$ and $Q_{n}$ which may 
be obtained by recognizing that $(i / \pi) Q_{n}(z)$ is the analytic representation of $P_{n} \chi$ where $\chi$ is the characteristic function of $[-1,1]$. Thus we have (see Bremermann [B-1, p. 48])

$$
Q_{n}(t+i 0)-Q_{n}(t-i 0)=-\pi i P_{n}(t), \quad-1<t<1 \text {. }
$$

This may also be found in Szegö [S-1, p. 79].

3. The main theorem. Let $P_{n}$ and $Q_{n}$ denote the Legendre functions of the first and second kind respectively and let $\left\{a_{n}\right\}$ be a sequence of complex numbers such that

$$
\left|a_{n}\right| \leqq M^{p}, \quad n=1,2, \cdots
$$

for some positive integers $M$ and $p$; let $f$ be the analytic function given for $|\xi|<1$ by $f(\xi)=\sum a_{n} \xi^{n}$. Then the series $\sum a_{n} P_{n}$ converges in $(-1,1)$ to $a$ distribution $g$, the series $\sum i \pi^{-1} a_{n} Q_{n}(z)$ converges for Im $z \neq 0$ to the analytic representation $\hat{g}(z)$ of $g$; the function $\hat{g}(z)$ is holomorphic in the plane cut along $[-1,1]$ and has a singular point at $z_{0}=\frac{1}{2}\left(\xi_{0}+1 / \xi_{0}\right)$ in $(-1,1)$ if and only if $f(\xi)$ has a singular point on the unit circle at $\xi_{0} \neq \pm 1$ and at $\bar{\xi}_{0}$.

We first prove that the series $\sum a_{n} P_{n}$ converges to a distribution in $(-1,1)$. Consider the function $F(t)=\sum a_{n} P_{n}(t)\left(n+\frac{1}{2}\right)^{-2 p-2}$ for $t \in[-1,1]$ and 0 for $t<-1$ and $t>1$. Then $F$ is continuous on $[-1,1]$, since the series converges uniformly. Since the Legendre differential operator $\mathscr{L}=\left\{\left(t^{2}-1\right) D^{2}+2 t D+\frac{1}{4}\right\}$ is continuous in $D^{\prime}$ (the space of distributions) and since uniform convergence implies convergence in $D^{\prime}$, we may define $g \in D^{\prime}$ by

$$
g=\mathscr{L}^{p+1} F=\sum a_{n}\left(n+\frac{1}{2}\right)^{-2 p-2} \mathfrak{L}^{p+1} P_{n} \chi .
$$

But $\mathscr{L}^{p+1}\left(P_{n} \chi\right)=\left(n+\frac{1}{2}\right)^{2 p+2} P_{n} \chi$ and therefore $g=\sum a_{n} P_{n} \chi$ or $g=\sum a_{n} P_{n}$ in $(-1,1)$.

The analytic representation $\hat{g}(z)$, since $g$ has compact support, is given by

$$
\hat{g}(z)=\frac{1}{2 \pi i}\langle g,\{1 /(t-z)\}\rangle, \quad \operatorname{Im} z \neq 0 .
$$

But $1 /(t-z)$ is $C^{\infty}$ on the real axis and hence its Legendre expansion converges uniformly on $[-1,1]$. Also the derivatives of its expansion converges uniformly to the corresponding derivatives of $1 /(t-z)$ on $[-1,1]$. By extending the Legendre polynomials from $[-1,1]$ to $C^{\infty}$ functions with support in $[-1-\epsilon, 1+\epsilon]$ and doing the same for 
$1 /(t-z)$, we may interpret this convergence to be in the sense of $D$ (the test function space). Thus we have

$$
\begin{aligned}
\left\langle g,\left\{\frac{1}{t-z}\right\}\right\rangle & =\left\langle g, \sum_{n=0}^{\infty} \int_{-1}^{1} \frac{P_{n}(t)}{t-z} d t\left(n+\frac{1}{2}\right) P_{n}\right\rangle \\
& =\sum_{n=0}^{\infty} \int_{-1}^{1} \frac{P_{n}(t)}{t-z} d t(n+1 / 2)\left\langle g, P_{n}\right\rangle \\
& =-\sum_{n=0}^{\infty} Q_{n}(z)(2 n+1)\left\langle g, P_{n}\right\rangle .
\end{aligned}
$$

But the coefficients of $g$ are given by

$$
\begin{aligned}
\left(n+\frac{1}{2}\right)\left\langle g, P_{n}\right\rangle & =\left(n+\frac{1}{2}\right)\left\langle\mathfrak{L}^{p+1} F, P_{n}\right\rangle=\left(n+\frac{1}{2}\right)\left\langle F, \mathfrak{L}^{p+1} P_{n}\right\rangle \\
& =\left(n+\frac{1}{2}\right)^{2 p+2}\left\langle F, P_{n}\right\rangle=a_{n} .
\end{aligned}
$$

Hence the analytic representation of $g$ is given by the series

$$
\hat{g}(z)=\sum \frac{i a_{n}}{\pi} Q_{n}(z)
$$

which converges for $z \notin[-1,1]$ since

$$
\begin{aligned}
\left|Q_{n}(z)\right| & =\frac{1}{2}\left|\int_{-1}^{1} \frac{P_{n}(t)}{t-z} d t\right| \\
& =\frac{1}{2}\left|\int_{-1}^{1} \frac{P_{n}(t)}{(n+1 / 2)^{2 n+2}} \mathfrak{L}^{P+1} \frac{1}{t-z} d t\right| \\
& \leqq \frac{M(z)}{(n+1 / 2)^{2 p+2}} .
\end{aligned}
$$

It remains to prove the statement about singular points. To do this we find an integral operator taking $f$ into $\hat{g}$ and another taking $\hat{g}$ into $f$.

Borrowing the technique of Gilbert and Howard [G-H-1], we can show that

$$
\hat{g}(z)=\frac{1}{2 \pi^{2}} \int_{C} K(z, \xi) f(\xi) \frac{d \xi}{\xi} \quad \text { for } \operatorname{Im} z \neq 0
$$

where $C$ is the contour $|\xi|=\rho<1$. Indeed, for Im $z$ sufficiently large the series defining $K(z, \xi)$ converges for $|\xi|=\rho<1$, and both $K(z, \xi)$ and $f(\xi)$ may be replaced by their series in the integral (11). Thus we have 


$$
\begin{aligned}
\int_{C} K(z, \xi) f(\xi) \frac{d \xi}{\xi} & =\int_{C} \sum_{n=0}^{\infty} Q_{n}(t) \xi^{-n} \sum_{n=0}^{\infty} a_{k} \xi^{k} \frac{d \xi}{\xi} \\
& =2 \pi i \sum_{n=0}^{\infty} a_{n} Q_{n}(z) .
\end{aligned}
$$

Again, by the Hadamard argument, $\hat{g}(z)$ has possible singularities only at those points which are simultaneously singularities of both $K(z, \xi)$ and $f(\xi)$; in this case (except for the points 1 and -1 ) the only such points are at a zero of $\xi_{0}^{2}-2 \xi_{0} z+1$ where $\xi_{0}$ is a singular point of $f$.

The integral operator taking $\hat{g}$ into $f$ is given by the integral

$$
\lim _{\epsilon \rightarrow 0} \int_{-1}^{+1} \frac{1-\xi^{2}}{\left(1-2 \xi t+\xi^{2}\right)^{3 / 2}}\{\hat{g}(t+i \epsilon)-\hat{g}(t-i \epsilon)\} d t, \quad|\xi|<1
$$

which may be expressed as a contour integral. If we deform the contour to exclude singular points $z_{0}$ of $\hat{g}$ and take $\xi$ sufficiently small, we may take the limit as $\epsilon \rightarrow 0$ and express it as an integral over a contour with end points at -1 and +1 and avoiding singularities of both factors. Again, the Hadamard argument is invoked as $|\xi|$ is allowed to approach 1 to conclude that the function given by each of these integrals has a possible singularity only at a zero of $\xi^{2}-2 \xi z_{0}+1$.

But we have that (12) is also given by

$$
\begin{aligned}
\left\langle g,\left\{\left(1-\xi^{2}\right)\left(1-2 \xi t+\xi^{2}\right)^{-3 / 2}\right\}\right\rangle & \\
& =\int_{-1-}^{1+} \sum_{n=0}^{\infty} \xi^{n} P_{n}(t)(2 n+1) \sum_{k=0}^{\infty} a_{k} P_{k}(t) d t \\
& =\sum_{n=0}^{\infty} a_{n} \xi^{n}=f(\xi),
\end{aligned}
$$

whence we conclude that $f(\xi)$ has its only possible singularities at that point.

4. Other orthogonal polynomials. Our result can be extended quid pro quo to Jacobi polynomials $\left\{P_{n}^{(\alpha, \beta)}\right\}$ for $\alpha, \beta>-1$. The analogs to the formulae (1), (2), (3), and (6) may be found again in Szegö [S-1, p. 75, 74, 69, and 79] respectively. The generating functions for $P_{n}^{(\alpha, \beta)}$ and $P_{n}$ both have singularities at the same point so that we can prove the lemma for $\sum Q_{n}^{(\alpha, \beta)}(z) \xi^{-n}$ with no change in the conclusion. However, we have no formula analogous to (5). But the corresponding series 


$$
\sum_{n=0}^{\infty} \frac{2 n+\alpha+\beta+1}{2^{\alpha+\beta+1}} \frac{\Gamma(n+1) \Gamma(n+\alpha+\beta+1)}{\Gamma(n+\alpha+1) \Gamma(n+\beta+1)} P_{n}^{(\alpha, \beta)}(t) \xi^{n}
$$

can be shown to converge to a function whose analytic continuation has a possible singular point only at $t=\frac{1}{2}(\xi+1 / \xi)$ by the analog to formula (3) and the Hadamard argument.

Again the same procedure can be used to show that $\sum a_{n} P_{n}^{(\alpha, \beta)}$ converges to a distribution $g$ on $(1,1)$. The only difference is that the Jacobi differential operator $\mathfrak{L}=\left(1-x^{2}\right) D^{2}+[\beta-\alpha-(\alpha+\beta+2) x] D$ is not selfadjoint. But it is always possible to find a continuous function $G$ such that $\left(\mathscr{L}^{*}\right)^{p} G=g$ where $\mathscr{L}^{*}$ is the conjugate operator and then to use this $G$ in place of $F$ in the paragraph preceding formula (10). The remainder of the proof presents no difficulties.

\section{REFERENCES}

[B-1] H. Bremermann, Distribution, complex variables, and Fourier transforms, Addison-Wesley, Reading, Mass., 1965.

[G-H-1] R. P. Gilbert and H. C. Howard, A generalization of a theorem of Nehari, Bull. Amer. Math. Soc. 72 (1966), 37-39.

[N-1] Z. Nehari, On singularities of Legendre expansions, J. Rational Mech. Anal. 5 (1956), 987-992.

[S-1] G. Szegö, Orthogonal polynomials, rev. ed., Amer. Math. Soc. Colloq. Publ., Vol. 23, Amer. Math. Soc., Providence, R. I., 1959.

University of Wisconsin, Milwaukee 